OPEN ACCESS

Edited by: Alberto Biondi,

Catholic University of the Sacred

Heart, Italy

Reviewed by:

Xiuying Xiao,

Shanghai JiaoTong University, China

Ming-Han Tsai,

National Yang-Ming University, Taiwan

*Correspondence:

Lin Shen

linshenpku@163.com

Jiafu Ji

jijiafu@hsc.pku.edu.cn

${ }^{\dagger}$ These authors share first authorship

Specialty section:

This article was submitted to Gastrointestinal Cancers,

a section of the journal

Frontiers in Oncology

Received: 29 September 2020

Accepted: 31 August 2021 Published: 23 September 2021

Citation:

Xie T, Peng Z, Liu Y, Zhang Z, Zhang X

Li J, Lu M, Gong J, Qi C, Ji J and

Shen L (2021) Clinicopathological

Characteristics and Response to

Chemotherapy in Treatment-Naive

Epstein-Barr Virus Associated Gastric

Cancer: A Retrospective Study.

Front. Oncol. 11:611676.

doi: 10.3389/fonc.2021.611676

\section{Clinicopathological Characteristics and Response to Chemotherapy in Treatment-Naive Epstein-Barr Virus Associated Gastric Cancer: A Retrospective Study}

\author{
Tong Xie ${ }^{1 \dagger}$, Zhi Peng ${ }^{1 \dagger}$, Yiqiang Liu ${ }^{2}$, Zhening Zhang ${ }^{1}$, Xiaotian Zhang ${ }^{1}$, Jian $\mathrm{Li}^{1}$, \\ Ming Lu ${ }^{1}$, Jifang Gong ${ }^{1}$, Changsong $\mathrm{Qi}^{1}$, Jiafu $\mathrm{Ji}^{3^{*}}$ and Lin Shen ${ }^{1 *}$
}

\begin{abstract}
1 Department of Gastrointestinal Oncology, Key Laboratory of Carcinogenesis and Translational Research (Ministry of Education), Peking University Cancer Hospital \& Institute, Beijing, China, ${ }^{2}$ Department of Pathology, Key Laboratory of Carcinogenesis and Translational Research (Ministry of Education), Peking University Cancer Hospital \& Institute, Beijing, China, ${ }^{3}$ Department of Gastrointestinal Surgery, Key Laboratory of Carcinogenesis and Translational Research (Ministry of Education), Peking University Cancer Hospital \& Institute, Beijing, China
\end{abstract}

Background: Epstein-Barr virus associated gastric cancer (EBVaGC) is a special subtype of gastric cancer. However, the perioperative treatment plan and the response to chemotherapy are still uncertain.

Methods: We retrospectively enrolled patients diagnosed with EBVaGC from March 2013 to July 2020 in Beijing Cancer Hospital. Clinicopathological characteristics were recorded. Disease-free survival (DFS) were then calculated, and variants affecting DFS were tested in a Cox proportional regression model.

Results: One hundred sixty consecutive patients were finally included in our study. Of the patients, 96.9\% had adenocarcinoma, while five had squamous cell carcinoma component. Most (70.9\%) of them were poorly differentiated. Prevalent programmed death-ligand 1 (PD-L1) (69\%) and minor HER-2 (3.8\%) expression were noticed; all of the patients were MMR proficient (pMMR) or microsatellite stable (MSS). Among 33 patients who experienced neoadjuvant therapy, the number of tumor regression grade (TRG) 1, TRG 2, and TRG 3 was 5, 16, and 12, respectively. Patients with advanced tumor stage and $\mathrm{T}$ stage showed poorer response. Thirty-one patients experienced first-line chemotherapy; ORR was 33.3\%, and DCR was 61.9\%. One hundred forty-seven patients underwent surgery, and 27 of them showed disease recurrence; the 3-year DFS rate was $71.0 \%$. Tumor stage, neoadjuvant chemotherapy, vascular invasion, and negative PD-L1 expression were associated with poorer DFS. Vascular invasion was the independent risk factor of DFS. Only seven patients reached OS with median follow-up time of 14 months. 
Conclusion: EBVaGC exhibits unique clinicopathological characteristics. Neoadjuvant chemotherapy may not be suitable for EBVaGC, and EBVaGC exhibited relatively poor response to chemotherapy.

Keywords: EBVaGC, clinicopathological characteristics, disease-free survival, objective response rate, chemotherapy

\section{INTRODUCTION}

Based on the results from multiomics sequencing, The Cancer Genome Atlas (TCGA) classified gastric cancer into four subtypes: microsatellite instability (MSI), Epstein-Barr virus (EBV) positive, genome stable (GS), and chromosome instability (CIN) (1). EBVassociated gastric cancer (EBVaGC) comprises $9 \%$ of gastric cancer approximately and exhibits massive lymphocyte infiltration, genome-wide hypermethylation, and prevalent programmed death-ligand 1 (PD-L1) expression $(2,3)$. Immunotherapy was then proposed for EBVaGC treatment basing on the histopathological features (4). However, the objective response rate (ORR) of PD-1/PD-L1 monotherapy was only $\sim 20 \%$ according to our previous investigation (5). The optimal treatment plan for EBVaGC is still unknown.

Surgery remains the key approach for gastric cancer treatment. Survival analysis of EBVaGC was conducted in several studies. However, the outcomes were inconsistent. Some studies revealed that EBV positivity correlated with a more favorable disease-free survival (DFS) $(6,7)$, while other studies found no significant difference in 3-year DFS or 5-year overall survival (OS) between EBVaGC and EBV-negative gastric cancer $($ EBVnGC) groups $(8,9)$. In addition, whether PD-L1 expression affects DFS outcome was controversial. Pereira et al. found no significant correlation between PD-L1 expression and 3 -year DFS rate $(73.9 \%$ vs. $73.2 \%, p=0.974)$ or 5 -year OS rate (72\% and $70.4 \%, p=0.908)$. Nevertheless, it was reported that intratumoral PD-L1 expression was significantly associated with lymph node metastasis $(p=0.012)$ and poorer DFS $(p=0.032)$ in another study (10). The impact of EBV infection to DFS and relating risk factors is still obscure.

On the other hand, in metastatic gastric cancer setting, the efficacy of chemotherapy was mildly described and was controversial. Corallo et al. reported that among six metastatic EBVaGC patients who received first-line chemotherapy, three patients showed CR and three patients showed PR, and the efficacy of chemotherapy was long lasting with median PFS of 31.9 months (11). The data were dramatically different from our previous understanding of palliative chemotherapy in stage IV gastric cancer. The favorable outcome might be due to the protective effect of high density of infiltrating lymphocyte. However, another study reported that the objective response rate (ORR) was only $29 \%$ in first-line chemotherapy (12). The response of EBVaGC to first-line chemotherapy still needs largescale clinical study to confirm.

The treatment strategy of EBVaGC is still uncertain. For locally advanced stage EBVaGC, whether preoperative chemotherapy adds survival benefit to EBVaGC and the data of pCR rate or TRG has not been reported yet. Furthermore, the efficacy of first-line chemotherapy to EBVaGC still need further exploration. Thus, to better understand the clinicopathological characteristics of EBVaGC and the response to chemotherapy, we retrospectively enrolled EBVaGC patients in our clinical center to investigate the treatment response of chemotherapy both in advanced stage and metastatic EBVaGC.

\section{METHODS}

\section{Population}

We retrospectively enrolled patients who were diagnosed with EBVaGC in Beijing Cancer Hospital from March 2013 to July 2020. Clinicopathological characteristics, such as gender, age, tumor stage, immunohistochemistry (IHC) results, and treatment plan, were recorded. Patients were staged according to the American Joint Committee on Cancer (AJCC), 7th edition, and RECIST 1.1 was used for tumor response evaluation. Both target and non-target lesions were evaluated; patients with only non-target lesions were judged as $\mathrm{CR}$, none $\mathrm{CR}$, none $\mathrm{PD}$, and $\mathrm{PD}$, and patients who had target lesions were divided into $\mathrm{CR}$, $\mathrm{PR}, \mathrm{SD}$, and PD.

\section{Pathological Examination}

Specimens obtained from surgery or biopsy were processed with formalin fixation and paraffin embedding. Tumor sections were subsequently evaluated by two experienced pathologists independently. Specimens were divided into intestinal, diffuse, and mixed according to Lauren classification. Tumors were classified into poorly, moderately-poorly, moderately, and highly differentiated based on the morphology of tumor cells after microscopic inspection. For patients who underwent neoadjuvant therapy, gastrectomy specimens were embedded, and tumor regression grade (TRG) was evaluated according to the percentage of viable tumor cell in the resected tumor. The criteria were adopted according to the China TRG (TRG $1=$ tumor cells completely disappear or very few highly regressive residues exist with obvious scarring and varying inflammation; TRG 2 = most tumor cells degenerate and necrotize with obvious stroma fibrosis and inflammation; TRG $3=$ absence of or slight necrosis and degeneration of tumor cells accompanied by mild stroma fibrosis and inflammation).

\section{IHC Staining}

MLH1 (GM002, Genetech), MSH2 (RED2, Genetech), MSH6 (EP49, Genetech), and PMS2 (EP51, Genetech) were stained for mismatch repair deficiency testing. Loss of nuclear staining in 
tumor cells was interpreted as MMR deficient (dMMR), otherwise MMR proficient (pMMR). 22C3 DAKO antibody was used for PD-L1 staining; combined positive score (CPS) was used for reporting. HER-2 [4B5, Roche (ULTRA)] was evaluated based on standard criteria; special situation such as heterogeneity or cytoplasm staining was recorded.

\section{EBV Detection}

EBV-encoded RNA was tested by in situ hybridization (Leica Biosystem), using unstained sections cut from paraffinembedded tumor blocks. Positive signals in tumor-cell nuclei together with negative signals in surrounding lymphocytes and normal tissue were considered to be positive result.

\section{MSI Testing}

MSI markers, namely, BAT-25, BAT-26, NR-21, NR-24, and MONO-27, were tested using PCR. Instabilities in two or more of them were categorized as MSI-high (MSI-H), instability in a single locus was categorized as MSI-low (MSI-L), and an absence of MSI in all the five markers was categorized as MSI-stable (MSS; GENTRON).

\section{Statistical Analysis}

In descriptive statistics, frequencies were calculated for nominal variables, and mean with \pm standard deviation (SD) or median \pm inter-quartile range was calculated for continuous variable. The chi-square test or Fisher's exact test was used for categorical variable, and t-tests were used for continuous variable to compare the difference among groups.

DFS was counted from the date of surgery to disease recurrence or death. DFS rate was obtained using Kaplan-Meier method. Factors, such as age, gender, Eastern Cooperative Oncology Group (ECOG) score, tumor stage, Lauren classification, and tumor marker level, were included for univariate Cox regression analysis. Variables that showed $p<0.15$ in univariate analysis were subsequently included in multivariate Cox proportional hazards models to explore the independent risk factors, in which stepwise method was used. Statistics analysis was performed using IBM SPSS version 25 (IBM, Armonk, NY, USA). Two-sided $p<0.05$ was considered as statistically significant.

\section{RESULTS}

\section{Clinicopathological Characteristics}

In total, 160 patients diagnosed with EBVaGC were finally included for our analysis. Of the patients, 85.8\% (139/160) were male. The median age was 56.5 years. 35\% (56/160) of the patients had positive drinking history, and 55\% (88/160) of them smoked. The clinicopathological characteristics are summarized in Table $\mathbf{1}$.

\section{Pathological Features}

Forty-two (26.2\%), 45 (28.1\%), 50 (31.2\%), and $23(14.4 \%)$ patients were staged I, II, III, and IV, respectively (Table 1). The numbers of tumors that were located in proximal stomach, gastric body, distal stomach, and remnant stomach were 38
TABLE 1 | Clinicopathological characteristics of EBVaGC.

\begin{tabular}{|c|c|}
\hline Character & N (\%) \\
\hline \multicolumn{2}{|l|}{ Gender } \\
\hline Male & $139(85.8)$ \\
\hline Female & $21(13.1)$ \\
\hline \multicolumn{2}{|l|}{ Age } \\
\hline Median & 56.5 \\
\hline \multicolumn{2}{|l|}{ Location } \\
\hline Proximal stomach & $38(23.7)$ \\
\hline Gastric body & $55(34.4)$ \\
\hline Distant stomach & $56(35)$ \\
\hline Remnant stomach & $11(6.9)$ \\
\hline \multicolumn{2}{|l|}{ Differentiation $(n=158)$} \\
\hline Poorly & $112(70.9)$ \\
\hline Moderately-poorly & $37(23.4)$ \\
\hline Moderately & $8(5.1)$ \\
\hline Highly & $1(0.6)$ \\
\hline \multicolumn{2}{|l|}{ Tumor stage } \\
\hline 1 & $42(26.2)$ \\
\hline$\|$ & $45(28.1)$ \\
\hline III & $50(31.2)$ \\
\hline IV & $23(14.4)$ \\
\hline \multicolumn{2}{|l|}{ T stage $(n=147)$} \\
\hline $\mathrm{T} 1$ & $26(17.7)$ \\
\hline T2 & $27(18.4)$ \\
\hline T3 & $47(32.0)$ \\
\hline $\mathrm{T} 4 \mathrm{a}$ & $40(27.2)$ \\
\hline $\mathrm{T} 4 \mathrm{~b}$ & $7(4.8)$ \\
\hline \multicolumn{2}{|l|}{ N stage $(n=148)$} \\
\hline NO & $71(48.0)$ \\
\hline $\mathrm{N} 1$ & $21(14.2)$ \\
\hline N2 & $24(16.2)$ \\
\hline $\mathrm{N} 3 \mathrm{a}$ & $18(12.2)$ \\
\hline N3b & $14(9.5)$ \\
\hline \multicolumn{2}{|c|}{ Lauren classification $(n=154)$} \\
\hline Intestinal & $40(26.0)$ \\
\hline diffuse & $43(27.9)$ \\
\hline Mixed & $71(46.1)$ \\
\hline \multicolumn{2}{|l|}{ HER-2 ( $n=158)$} \\
\hline 0 & 85 (53.8) \\
\hline $1+$ & $51(32.2)$ \\
\hline $2+$ & $16(10.1)$ \\
\hline $3+$ & $6(3.8)$ \\
\hline \multicolumn{2}{|l|}{ PD-L1 (n=100) } \\
\hline Positive & $69(69)$ \\
\hline Negative & $31(31)$ \\
\hline \multicolumn{2}{|c|}{ Metastatic sites $(n=31)$} \\
\hline Liver & $9(20.0 \%)$ \\
\hline Peritoneal & $13(41.9 \%)$ \\
\hline distant lymph node & $22(71.0 \%)$ \\
\hline
\end{tabular}

(23.7\%), 55 (34.4\%), $56(35 \%)$, and $11(6.9 \%)$, respectively. Nearly all the patients $(96.9 \%, 155 / 160)$ had gastric adenocarcinoma, while two patients were diagnosed as having squamous cell carcinoma, and three patients had adenosquamous cell carcinoma after inspection and IHC staining confirmation. Tumors were divided into poorly, moderately-poorly, moderately, and highly differentiated base on microscopic morphology, and the numbers were 112 (70.9\%), 37 (23.4\%), 8 (5.1\%), and $1(0.6 \%)$, respectively. One hundred fifty-four patients with biopsy or surgery samples were included for Lauren classification, and 40 (26.0\%), 43 (27.9\%), and $71(46.1 \%)$ of the 
patients were classified as intestinal, diffuse, and mixed types of gastric cancer.

\section{Molecular Features}

One hundred patients had definite PD-L1 results, $69 \%$ of the patients were positive, and the median CPS was 10 . There was no difference in tumor stage $\left(\chi^{2}=0.215, p=0.898\right)$, T stage $\left(\chi^{2}=\right.$ $0.850, p=0.860)$, or $\mathrm{N}$ stage $\left(\chi^{2}=0.215, p=0.741\right)$ between PD-L1-positive and PD-L1-negative groups. Only 6 (3.8\%) patients showed HER-2 (3+) among 158 patients who had confirmed results, and the number of patients who were HER2 (0), HER-2 (1+), HER-2 (2+) was 85 (53.8\%), 51 (32.3\%), and $16(10.1 \%)$, respectively. All of the patients with results showed pMMR and MSS.

\section{Response to Chemotherapy in Treatment-Naive EBVaGC}

Among the patients who underwent surgery, 33 patients experienced neoadjuvant therapy; the clinicopathological information is shown in Supplementary Table S1. All of the patients received $\mathrm{R} 0$ resection. The number of patients determined as TRG 1, TRG 2, and TRG 3 was 5 (15.2\%), $16(48.5 \%)$, and $12(36.4 \%)$, respectively. Two patients who received pembrolizumab combined with chemotherapy showed TRG 3. Tumor regression was statistically poorer in patients with advanced stage $(p=0.027)$, especially T stage $(p=0.007)$ (Supplementary Table S2). One patient was confirmed as pathological CR (pCR) after surgery, and the pCR rate was $3.03 \%$. There was no difference in Ki-67 or PD-L1 CPS in different TRG groups (Supplementary Figure S1).

Thirty-one patients received first-line chemotherapy. Among them, 9 patients showed liver metastasis, 13 experienced peritoneal metastasis, and 22 patients distant lymph node metastasis; the detailed information is shown in Supplementary Table S3. Twenty-eight patients had definite response evaluation results based on RECIST 1.1. Seven patients showed PR, six patients showed SD, seven patients who did not have target lesion were none CR none PD, and eight patients showed PD. The ORR was $33.3 \%$, and DCR was $61.9 \%$. There was no difference in PD-L1 $(p=0.58)$ or Ki-67 $(p=0.58)$ according to tumor response (Supplementary Figure S2). The median followup time was 14 months; only seven patients reached OS.

\section{DFS}

One hundred forty-seven patients underwent surgery, with $94.5 \%$ of them radical; eight patients received palliative surgery to reduce tumor burden when disease was stable. Among the patients who received radical surgery, 27 patients showed disease recurrence with median follow-up time of 20.7 months; the 3 -year DFS rate was $71.0 \%$ (Figure 1). The median DFS was not reached. The results of univariant Cox regression analysis are shown in Table 2. Patients with advanced tumor stage $(p=0.003)$, T stage $(p=0.002), \mathrm{N}$ stage $(p=0.002)$, negative PD-L1 expression $(p=0.048)$, and vascular invasion $(p=0.013)$, exhibited poorer DFS (Figures 2B-F). In multivariant Cox regression model, vascular invasion $(p=0.013)$ was the independent risk factor of DFS (Table 2).

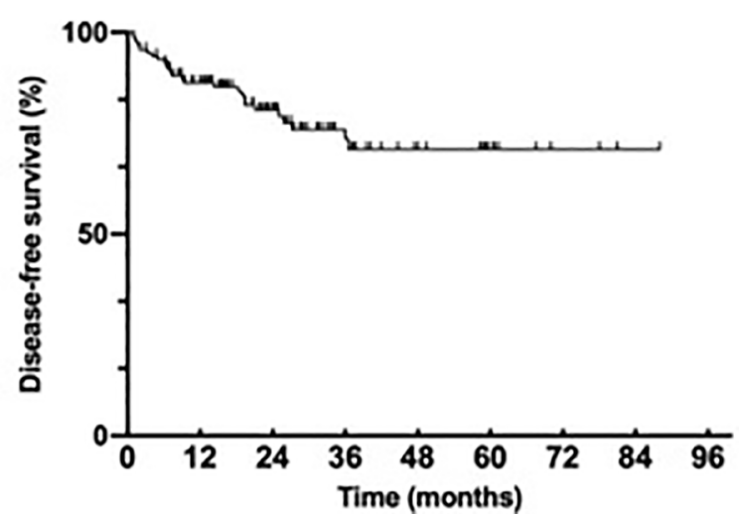

FIGURE 1 | Disease free survival of EBVaGC.

To figure out the impact of neoadjuvant chemotherapy to DFS, we compared the DFS in patients who were clinically staged II-III. There was no difference in Tumor stage $\left(\chi^{2}=0.836, p=0.469\right), \mathrm{T}$ stage $\left(\chi^{2}=3.039, p=0.233\right), \mathrm{N}$ stage $\left(\chi^{2}=5.852, p=0.114\right)$ between patients who underwent neoadjuvant chemotherapy and those who did not. DFS was significantly poorer in neoadjuvant chemotherapy group $(p=0.010)$ (Figure 2A).

\section{DISCUSSION}

Our retrospective study investigated the clinicopathological characteristics and the DFS and associated risk factors of EBVaGC in detail. To our knowledge, this is the first study that adequately reported the negative effect of neoadjuvant chemotherapy to DFS in EBVaGC. In addition, we reported the even poorer response to chemotherapy in treatment-naive EBVaGC patients.

EBVaGC exhibits unique clinicopathological characteristics. Our study confirmed the features, such as the gender discrepancy and PD-L1 expression. Moreover, we found that HER-2 was mildly expressed in EBVaGC; the proportion of patients diagnosed with HER-2 (3+) (3.8\%) was lower than average. As we all known, the pathogenesis of EBVaGC correlates with genome-wide hypermethylation, which is non-random; for example, no study reported MLH1 methylation until now, and the gene alteration of EBVaGC exhibits homogeneity (13). The expression of HER-2 may be deregulated due to methylation during the pathogenesis. Moreover, we found that five patients had squamous cell component after pathology inspection and diagnosed as squamous cell carcinoma or adenosquamous cell carcinoma, which is very rare in gastric cancer with an incidence of $0.04 \%$ $0.07 \%$. Cases of gastric cancer with squamous cell carcinoma with positive EBER-ISH result have also been reported (14). The etiology of primary gastric squamous cell carcinoma (PGSCC) is still uncertain; theories such as ectopic squamous epithelium, squamous metaplasia, or differentiation were proposed (15). The infection of EBV may participate in the process. Among the five patients who had squamous cell component, two patients reported no recurrence after surgery after 60.7 and 37.7 months 
TABLE 2 | Results from Cox regression analysis for DFS.

\begin{tabular}{|c|c|c|c|c|c|}
\hline Variants & $\beta$ & SE & HR & $95 \% \mathrm{Cl}$ & $\mathbf{P}$ \\
\hline Neoadjuvant chemotherapy ${ }^{1}$ & -1.019 & 0.393 & 0.361 & $0.167-0.781$ & $0.010^{*}$ \\
\hline Stage & 0.840 & 0.280 & 2.317 & $1.338-4.010$ & $0.003^{*}$ \\
\hline T stage & 0.701 & 0.222 & 2.015 & $1.304-3.113$ & $0.002^{*}$ \\
\hline $\mathrm{N}$ stage & 0.481 & 0.157 & 1.617 & $1.188-2.202$ & $0.002^{*}$ \\
\hline Lauren classification & -0.131 & 0.241 & 0.877 & $0.547-1.407$ & 0.587 \\
\hline Perineural invasion & -0.497 & 0.378 & 0.609 & $0.290-1.277$ & 0.189 \\
\hline PD-L1 expression² & 1.024 & 0.518 & 2.784 & $1.008-7.683$ & $0.048^{\star}$ \\
\hline \multicolumn{6}{|c|}{ Multivariant Cox regression analysis } \\
\hline Vascular invasion & -1.094 & 0.439 & 0.335 & $0.142-0.792$ & $0.013^{*}$ \\
\hline
\end{tabular}

* stands for statistical significance.

${ }^{1}$ Neoadjuvant chemotherapy was excluded from multivariant analysis, as it was analyzed only in part of the patients.

${ }^{2}$ PD-L1 was excluded from multivariant analysis due to missing values.

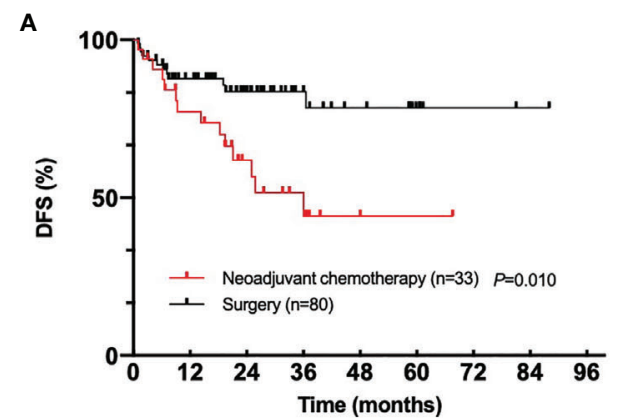

C

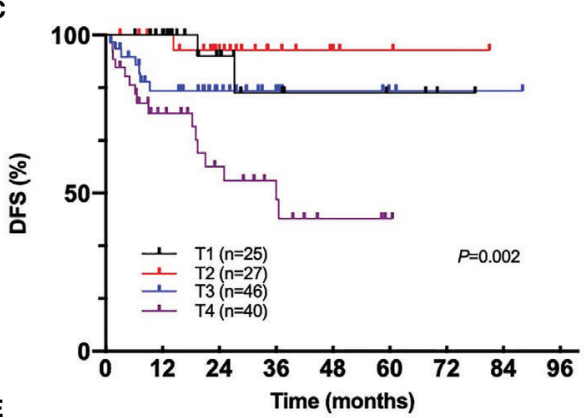

E

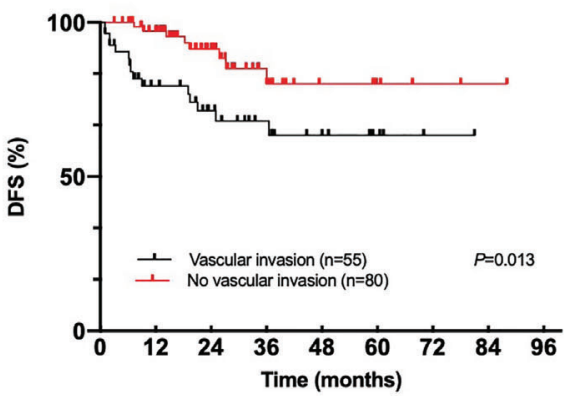

B

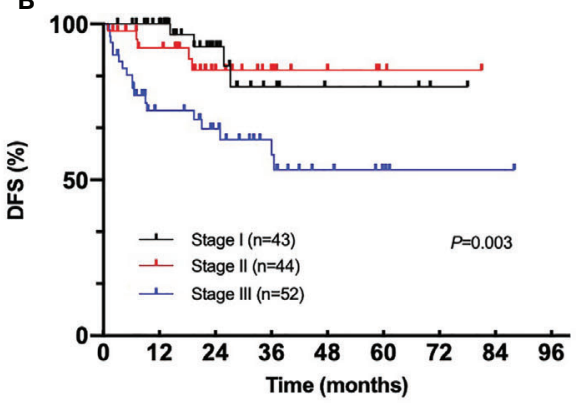

D

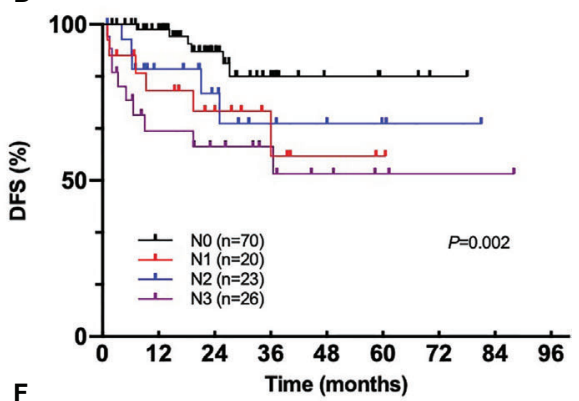

F

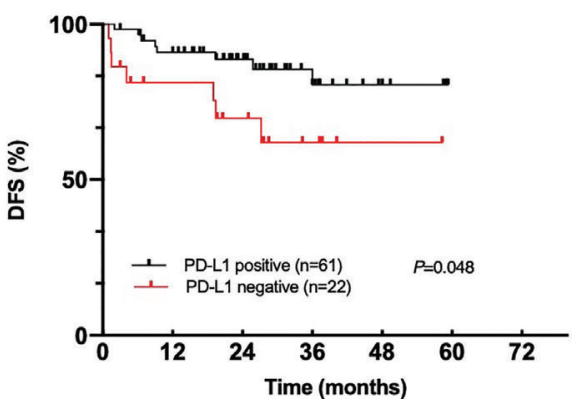

FIGURE 2 | DFS in different groups of EBVaGC: (A), neoadjuvant chemotherapy; (B), tumor stage; (C), T stage; (D), N stage; (E), vascular invasion; (F), PD-L1 expression. 
follow-up, one patient with PD-L1 CPS 80 showed TRG 1 after paclitaxel-based neoadjuvant chemotherapy and PR to first-line chemotherapy, and one patient showed SD after first-line chemotherapy. It seems that there was no difference in tumor response or survival in EBVaGC with squamous cell component compared with adenocarcinoma, which may need further study to confirm.

Our study found that among 147 patients who underwent surgery, only 27 of them showed recurrence; the 3-year DFS rate was $71.0 \%$. RESOLVE study, which was conducted mainly in our medical center and published in the LANCET Oncol recently, reported that the 3-year DFS rate in adjuvant CapeOX, adjuvant SOX, and perioperative SOX group was $51.1 \%, 56.5 \%$, and $59.4 \%$, respectively (16). Interestingly, different from the results from RESOLVE, in which SOX neoadjuvant arm showed superior DFS compared with the surgery plus XELOX arm (HR = 0.79 ; 95\% CI, 0.62-0.99, $p=0.045$ ), our study demonstrated the opposite conclusion, in which neoadjuvant chemotherapy shortened the DFS in EBVaGC patients. Similarly, subgroup analysis of MAGIC study demonstrated that dMMR or MSI-H was associated with a negative prognostic effect in patients treated with chemotherapy. None of the patients in MAGIC study had good pathological response to chemotherapy, while $14 \%$ of pMMR patients exhibited TRG 1 or TRG 2 (Mandard tumor regression grading system). An individual meta-analysis subsequently confirmed the negative effect of perioperative chemotherapy to DFS and OS, pooling the data from MAGIC, CLASSIC, ARTIST, and ITACA-S trials (17). EBVaGC and MSI-H gastric cancer exhibited the same pattern of response to neoadjuvant chemotherapy. What is consistent with dMMR/MSI-H and EBVaGC is the massive infiltration of lymphocyte, especially CD8+ $\mathrm{T}$ cells. The underling mechanism of inferior effects to both EBVaGC and dMMR/MSI-H patients may due to the disruption of protective microenvironment by chemotherapy or the tumor cell owned different response mechanism to chemotherapy due to the special genetic or epigenetic changes.

$\mathrm{PD}-\mathrm{L} 1$ is a very common checkpoint constitutively expressed on the surface of normal cells. The activation of PD-1 pathway leads to T-cell exhaustion. Not only the normal stromal tissue but also tumor cells could express PD-L1 in the tumor microenvironment, escaping the attack from cytotoxic $\mathrm{T}$ cell. However, the impact of PD-L1 expression to the survival of EBVaGC was controversial. For DFS, Seo et al. found that intratumoral PD-L1 expression was associated with poorer DFS ( $\mathrm{HR}=12.085$; 95\% CI, 2.013-72.559, $p$ $=0.006)(10)$. Sundar et al. divided EBVaGC into PD-L1 $1_{\text {low }}$ and PD$\mathrm{L1}_{\text {high }}$ groups and reported that EBVaGC with high PD-L1 expression level was associated with more favorable DFS (HR = 5.03; 95\% CI, 0.97-25.92; $p=0.032$ ) (18). Furthermore, no discrepancy in DFS with regard to PD-L1 expression was also reported in another study (19). When we look back into the data of the whole gastric cancer, the prognostic value of PD-L1 in OS is also debatable (20-22). Excluding other confounding factors such as tumor stage, $\mathrm{T}$ stage, or $\mathrm{N}$ stage, we found that PD-L1 expression was associated with longer DFS. The complicated PD-L1 expression effects to survival may due to following reasons: (1) antibody clonal used for PD-L1 staining different across studies, (2) no standard criteria and cutoffs for assessing positivity, (3) the temporal and intratumor heterogeneity in EBVaGC, and (4) the races of enrolled patients and species of infected virus. Moreover, as for patients who received neoadjuvant or first-line chemotherapy, there was no difference in PD-L1 expression level among different responsive groups. The predicting value of PD-L1 in survival but not efficacy was interesting, which may due to the relatively small sample size in neoadjuvant or first-line chemotherapy on the one hand, but could also be interpreted as the chemotherapy-insensitive but protective inflamed microenvironment.

Although EBVaGC was demonstrated to have lower T stage (7), we found that lymph node was still the most often metastatic site. With regard to EBVnGC, previous studies reported that the perineum turned out to be the most often recurrent site $(23,24)$; the divergence indicates that the metastasis of EBVaGC might rely on a unique biological mechanism, which may need further study to investigate. In our study, only one patient showed pCR, and the pCR rate was only $3.03 \%$. Another study that was also conducted in our medical center in an EBVnGC population reported that the pCR rate was $11.8 \%$, and the percent of patients who reached TRG0 or TRG1 was $20.6 \%$, while only $15.1 \%$ of our patients exhibited TRG1 (23). A larger sample size study, including 473 gastric cancer patients who received neoadjuvant chemotherapy, exhibited a pCR rate of $5.9 \%$ (25). The pCR rate of $3.03 \%$ in our study is far behind from other reported data. EBVaGC may be relatively less sensitive to chemotherapy. In contrast with previously reported data on EBVaGC first-line chemotherapy, which reported $100 \%$ ORR and long-lasting effects, we found that the ORR in our study was $33.3 \%$, which is even lower than the data of the whole gastric cancer group (24). Qiu et al. reported an even lower ORR of EBVaGC in another retrospective study (12). Similarly, as mentioned above, chemotherapy may disrupt the protective effect of the infiltrated CD8+ T cells, thus shortening the DFS. The ORR in our study might be due to the same reason.

Our study provided sufficient evidence to the clinicopathological features of EBVaGC; however, as most of the patients were in an early or advanced stage and experienced radical surgery, the sample size of patients who underwent first-line chemotherapy was relatively small. We still need further larger-scale study to confirm the findings in the future. As our study was a retrospective study, part of the information was incomplete, for example, the exact stromal or tumoral PD-L1 expression. Owing to the favorable OS of $\mathrm{EBVaGC}$ and adequate later-line treatment, like immunotherapy, only seven patients reached OS, and the analysis of OS was skipped. Prospective observation is currently in progress.

Herein, we summarized the clinicopathological features of EBVaGC and reported the DFS and related risk factors in detail, along with the response of first-line chemotherapy of EBVaGC. We primarily reported the negative effect of neoadjuvant chemotherapy to DFS and the prognostic value of PD-L1 to survival. Although immunotherapy was proposed for the treatment of EBVaGC, both of the patients who experienced immunotherapy in our study showed TRG 3, and chemotherapy seems to have similar efficacy compared with single agent immunotherapy. The exact treatment landscape of EBVaGC is still uncertain. Combined immunotherapy seems to have very promising preliminary results; however, the combination regimens and the place of chemotherapy still need further exploration. 


\section{DATA AVAILABILITY STATEMENT}

The raw data supporting the conclusions of this article will be made available by the authors, without undue reservation.

\section{ETHICS STATEMENT}

Ethical review and approval was not required for the study on human participants in accordance with the local legislation and institutional requirements. Written informed consent was not provided because This is a retrospective study, ethics review was not required by our committee.

\section{AUTHOR CONTRIBUTIONS}

LS and JJ designed this investigation. TX and ZP analyzed the data and completed the manuscript. ZZ revised the paper. YL provided the pathological information of the patients. JL, XZ,

\section{REFERENCES}

1. The Cancer Genome Atlas. Comprehensive Molecular Characterization of Gastric Adenocarcinoma. Nature (2014) 513(7517):202-9. doi: 10.1038/nature13480

2. Ignatova E, Seriak D, Fedyanin M, Tryakin A, Pokataev I, Menshikova S, et al. Epstein-Barr Virus-Associated Gastric Cancer: Disease That Requires Special Approach. Gastric Cancer (2020) 23(6):951-60. doi: 10.1007/s10120-020-01095-Z

3. Naseem M, Barzi A, Brezden-Masley C, Puccini A, Berger MD, Tokunaga R, et al. Outlooks on Epstein-Barr Virus Associated Gastric Cancer. Cancer Treat Rev (2018) 66:15-22. doi: 10.1016/j.ctrv.2018.03.006

4. Zhang Z, Xie T, Zhang X, Qi C, Shen L, Peng Z. Immune Checkpoint Inhibitors for Treatment of Advanced Gastric or Gastroesophageal Junction Cancer: Current Evidence and Future Perspectives. Chin J Cancer Res (2020) 32(3):287-302. doi: 10.21147/j.issn.1000-9604.2020.03.02

5. Xie T, Liu Y, Zhang Z, Zhang X, Gong J, Qi C, et al. Positive Status of EpsteinBarr Virus as a Biomarker for Gastric Cancer Immunotherapy: A Prospective Observational Study. J immunotherapy (Hagerstown Md 1997) (2020) 43 (4):139-44. doi: 10.1097/CJI.0000000000000316

6. Ramos M, Pereira MA, Amorim LC, de Mello ES, Faraj SF, Ribeiro U, et al. Gastric Cancer Molecular Classification and Adjuvant Therapy: Is There a Different Benefit According to the Subtype? J Surg Oncol (2020) 121 (5):804-13. doi: 10.1002/jso.25792

7. van Beek J, zur Hausen A, Klein Kranenbarg E, van de Velde CJ, Middeldorp JM, van den Brule AJ, et al. EBV-Positive Gastric Adenocarcinomas: A Distinct Clinicopathologic Entity With a Low Frequency of Lymph Node Involvement. J Clin Oncol (2004) 22(4):664-70. doi: 10.1200/JCO.2004.08.061

8. Pereira MA, Ramos M, Faraj SF, Dias AR, Yagi OK, Zilberstein B, et al. Clinicopathological and Prognostic Features of Epstein-Barr Virus Infection, Microsatellite Instability, and PD-L1 Expression in Gastric Cancer. J Surg Oncol (2018) 117(5):829-39. doi: 10.1002/jso.25022

9. Fang WL, Chen $\mathrm{MH}$, Huang $\mathrm{KH}$, Lin $\mathrm{CH}$, Chao $\mathrm{Y}$, Lo SS, et al. The Clinicopathological Features and Genetic Alterations in Epstein-Barr VirusAssociated Gastric Cancer Patients After Curative Surgery. Cancers (Basel) (2020) 12(6):1517. doi: 10.3390/cancers12061517

10. Seo AN, Kang BW, Kwon OK, Park KB, Lee SS, Chung HY, et al. Intratumoural PD-L1 Expression Is Associated With Worse Survival of Patients With Epstein-Barr Virus-Associated Gastric Cancer. Br J Cancer (2017) 117(12):1753-60. doi: 10.1038/bjc.2017.369

11. Corallo S, Fucà G, Morano F, Salati M, Spallanzani A, Gloghini A, et al. Clinical Behavior and Treatment Response of Epstein-Barr Virus-Positive and ML provided conception advice to the manuscript. CQ and JG provided statistics analysis advice. All authors contributed to the article and approved the submitted version.

\section{FUNDING}

Clinical Medicine Plus X-Young Scholars Project, Peking University: the Fundamental Research Funds for the Central Universities (PKU2021LCXQ016), the third round of public welfare development and reform pilot projects of Beijing Municipal Medical Research Institutes (Beijing Medical Research Institute, 2019-1).

\section{SUPPLEMENTARY MATERIAL}

The Supplementary Material for this article can be found online at: https://www.frontiersin.org/articles/10.3389/fonc.2021. 611676/full\#supplementary-material
Metastatic Gastric Cancer: Implications for the Development of Future Trials. Oncologist (2020) 25(9):780-6. doi: 10.1634/theoncologist.2020-0037

12. Qiu MZ, He CY, Yang DJ, Zhou DL, Zhao BW, Wang XJ, et al. Observational Cohort Study of Clinical Outcome in Epstein-Barr Virus Associated Gastric Cancer Patients. Ther Adv Med Oncol (2020) 12:1758835920937434. doi: $10.1177 / 1758835920937434$

13. Matsusaka K, Kaneda A, Nagae G, Ushiku T, Kikuchi Y, Hino R, et al. Classification of Epstein-Barr Virus-Positive Gastric Cancers by Definition of DNA Methylation Epigenotypes. Cancer Res (2011) 71(23):7187-97. doi: 10.1158/0008-5472.CAN-11-1349

14. Takita J, Kato H, Miyazaki T, Nakajima M, Fukai Y, Masuda N, et al. Primary Squamous Cell Carcinoma of the Stomach: A Case Report With Immunohistochemical and Molecular Biologic Studies. Hepatogastroenterology (2005) 52(63):969-74.

15. Beattie M, Mansour R, Thigpin D, Haus C. Metastatic Primary Gastric Squamous Cell Carcinoma: An Uncommon Presentation of a Rare Malignancy. Case Rep Gastrointest Med (2019) 2019:5305023. doi: 10.1155/ 2019/5305023

16. Zhang X, Liang H, Li Z, Xue Y, Wang Y, Zhou Z, et al. Perioperative or Postoperative Adjuvant Oxaliplatin With S-1 Versus Adjuvant Oxaliplatin With Capecitabine in Patients With Locally Advanced Gastric or GastroOesophageal Junction Adenocarcinoma Undergoing D2 Gastrectomy (RESOLVE): An Open-Label, Superiority and non-Inferiority, Phase 3 Randomised Controlled Trial. Lancet Oncol (2021) 22(8):1081-92. doi: 10.1016/S1470-2045(21)00297-7

17. Pietrantonio F, Miceli R, Raimondi A, Kim YW, Kang WK, Langley RE, et al. Individual Patient Data Meta-Analysis of the Value of Microsatellite Instability As a Biomarker in Gastric Cancer. J Clin Oncol (2019) 37 (35):3392-400. doi: 10.1200/JCO.19.01124

18. Sundar R, Qamra A, Tan ALK, Zhang S, Ng CCY, Teh BT, et al. Transcriptional Analysis of Immune Genes in Epstein-Barr VirusAssociated Gastric Cancer and Association With Clinical Outcomes. Gastric Cancer (2018) 21(6):1064-70. doi: 10.1007/s10120-018-0851-9

19. Kawazoe A, Kuwata T, Kuboki Y, Shitara K, Nagatsuma AK, Aizawa M, et al. Clinicopathological Features of Programmed Death Ligand 1 Expression With Tumor-Infiltrating Lymphocyte, Mismatch Repair, and Epstein-Barr Virus Status in a Large Cohort of Gastric Cancer Patients. Gastric Cancer (2017) 20(3):407-15. doi: 10.1007/s10120-016-0631-3

20. Saito R, Abe H, Kunita A, Yamashita H, Seto Y, Fukayama M. Overexpression and Gene Amplification of PD-L1 in Cancer Cells and PD-L1(+) Immune Cells in 
Epstein-Barr Virus-Associated Gastric Cancer: The Prognostic Implications. Mod Pathol (2017) 30(3):427-39. doi: 10.1038/modpathol.2016.202

21. Ma T, Wu ZJ, Xu H, Wu CH, Xu J, Peng WR, et al. Nomograms for Predicting Survival in Patients With Metastatic Gastric Adenocarcinoma Who Undergo Palliative Gastrectomy. BMC Cancer (2019) 19(1):852. doi: 10.1186/s12885019-6075-5

22. Takahashi R, Ohashi M, Kano Y, Ida S, Kumagai K, Nunobe S, et al. Timing and Site-Specific Trends of Recurrence in Patients With Pathological Stage II or III Gastric Cancer After Curative Gastrectomy Followed by Adjuvant S-1 Monotherapy. Gastric Cancer (2019) 22(6):1256-62. doi: 10.1007/s10120019-00953-9

23. Fu J, Tang L, Li ZY, Li XT, Zhu HF, Sun YS, et al. Diffusion Kurtosis Imaging in the Prediction of Poor Responses of Locally Advanced Gastric Cancer to Neoadjuvant Chemotherapy. Eur J Radiol (2020) 128:108974. doi: 10.1016/ j.ejrad.2020.108974

24. Lordick F, Lorenzen S, Yamada Y, Ilson D. Optimal Chemotherapy for Advanced Gastric Cancer: Is There a Global Consensus? Gastric Cancer (2014) 17(2):213-25. doi: 10.1007/s10120-013-0297-Z

25. Li Z, Wang Y, Shan F, Ying X, Wu Z, Xue K, et al. ypTNM Staging After Neoadjuvant Chemotherapy in the Chinese Gastric Cancer Population: An
Evaluation on the Prognostic Value of the AJCC Eighth Edition Cancer Staging System. Gastric Cancer (2018) 21(6):977-87. doi: 10.1007/s10120-018-0830-1

Conflict of Interest: The authors declare that the research was conducted in the absence of any commercial or financial relationships that could be construed as a potential conflict of interest.

Publisher's Note: All claims expressed in this article are solely those of the authors and do not necessarily represent those of their affiliated organizations, or those of the publisher, the editors and the reviewers. Any product that may be evaluated in this article, or claim that may be made by its manufacturer, is not guaranteed or endorsed by the publisher.

Copyright (C) 2021 Xie, Peng, Liu, Zhang, Zhang, Li, Lu, Gong, Qi, Ji and Shen. This is an open-access article distributed under the terms of the Creative Commons Attribution License (CC BY). The use, distribution or reproduction in other forums is permitted, provided the original author(s) and the copyright owner(s) are credited and that the original publication in this journal is cited, in accordance with accepted academic practice. No use, distribution or reproduction is permitted which does not comply with these terms. 